\title{
Purpura Fulminans and Late Onset Group B Streptococcal Sepsis in a Premature Twin
}

\author{
Avanikkha Elayappen, MD ${ }^{1}$ Sunil K. Jain, $\mathrm{MD}^{1} \quad$ Michael J. Loeffelholz, $\mathrm{PhD}^{2} \quad$ Janak Patel, MD \\ ${ }^{1}$ Department of Pediatrics, University of Texas Medical Branch, \\ Galveston, Texas \\ 2 Department of Pathology, University of Texas Medical Branch, \\ Address for correspondence Sunil K. Jain, MD, Division of \\ Neonatology, Department of Pediatrics, 301 University Boulevard, \\ Galveston, TX 77555-0526 (e-mail: skjain@utmb.edu).
} Galveston, Texas

Am J Perinatol Rep 2014;4:e69-e72.

\begin{abstract}
Keywords

- purpura fulminans

- GBS

- neonate

- meningitis

- coagulation

- serotype la

Introduction: Purpura fulminans (PF) is a skin manifestation due to hemorrhagic infarction caused by intravascular thrombosis secondary to bacterial infections or deficiency of anticoagulants such as protein $\mathrm{C}$ and protein S. Neonatal PF is a rare but potentially disabling disorder associated with a high mortality and severe long term morbidity in those who survive.

Case description: We report a case of a premature infant who developed extensive PF due to late onset group B streptococcus sepsis. Despite early identification and initiation of antibiotic therapy in our patient, PF progressed rapidly, leading to autoamputation of fingers and toes and severe brain injury.

Conclusion: In conclusion, our case highlights the severe sequelae of PF due to late onset GBS sepsis in a premature infant.
\end{abstract}

Purpura fulminans (PF) in the neonate is a rare but potentially disabling illness of acute onset associated with high mortality and long-term morbidity. Its skin manifestations are due to hemorrhagic infarction and gangrenous necrosis caused by intravascular thrombosis. It is most commonly associated with bacterial infections or deficiency of anticoagulants such as protein $\mathrm{C}$ and protein $\mathrm{S}$. Infection activates the coagulation cascade leading to consumptive coagulopathy and development of microthrombi, which in turn lead to tissue necrosis. While full-term neonates with PF have been described, to our knowledge, we are reporting the first case of severe PF secondary to late onset group B streptococcus (GBS) sepsis in an extremely premature infant.

\section{Case Report}

A 1,390 g male infant, larger of discordant Di/Di twins, was born at 27-week gestation via cesarean section due to preeclampsia and preterm labor. Pregnancy of the mother was complicated by gonorrhea and chlamydia infections, which were treated adequately. The mother was negative for rapid plasma reagin, hepatitis B surface antigen, and HIV antibody. A rectovaginal swab culture at 19 -week gestation was positive for GBS. She was treated with intravenous (IV) ampicillin every 4 hours from 36 hours before delivery. The amniotic membranes were ruptured at the time of delivery. The Apgar scores were 7 and 8 at 1 and 5 minutes, respectively. At birth, the infant received intratracheal surfactant and was extubated immediately to continuous positive airway pressure. Infant was started on IV ampicillin and gentamicin until 48 hours negative blood culture. He was tolerating oral feeding (expressed breast milk) and gaining weight. A routine head ultrasound on day 7 was normal.

On day 25, the infant's condition deteriorated with increased respiratory distress, hypotension, tachycardia, and skin mottling. Sepsis workup was performed which included complete blood count (CBC), blood culture, urine culture, and lumbar puncture for cerebrospinal fluid (CSF) culture. The infant was managed with mechanical ventilation and begun on IV antibiotics (vancomycin $20 \mathrm{mg} / \mathrm{kg} /$ dose every 18 hours received

January 14, 2014 accepted after revision

March 20, 2014

published online

June 10, 2014
DOI http://dx.doi.org/ 10.1055/s-0034-1376186. ISSN 2157-6998.
Copyright $\odot 2014$ by Thieme Medical Publishers, Inc., 333 Seventh Avenue, New York, NY 10001, USA. Tel: +1(212) 584-4662.
License terms

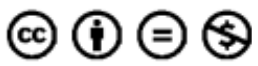




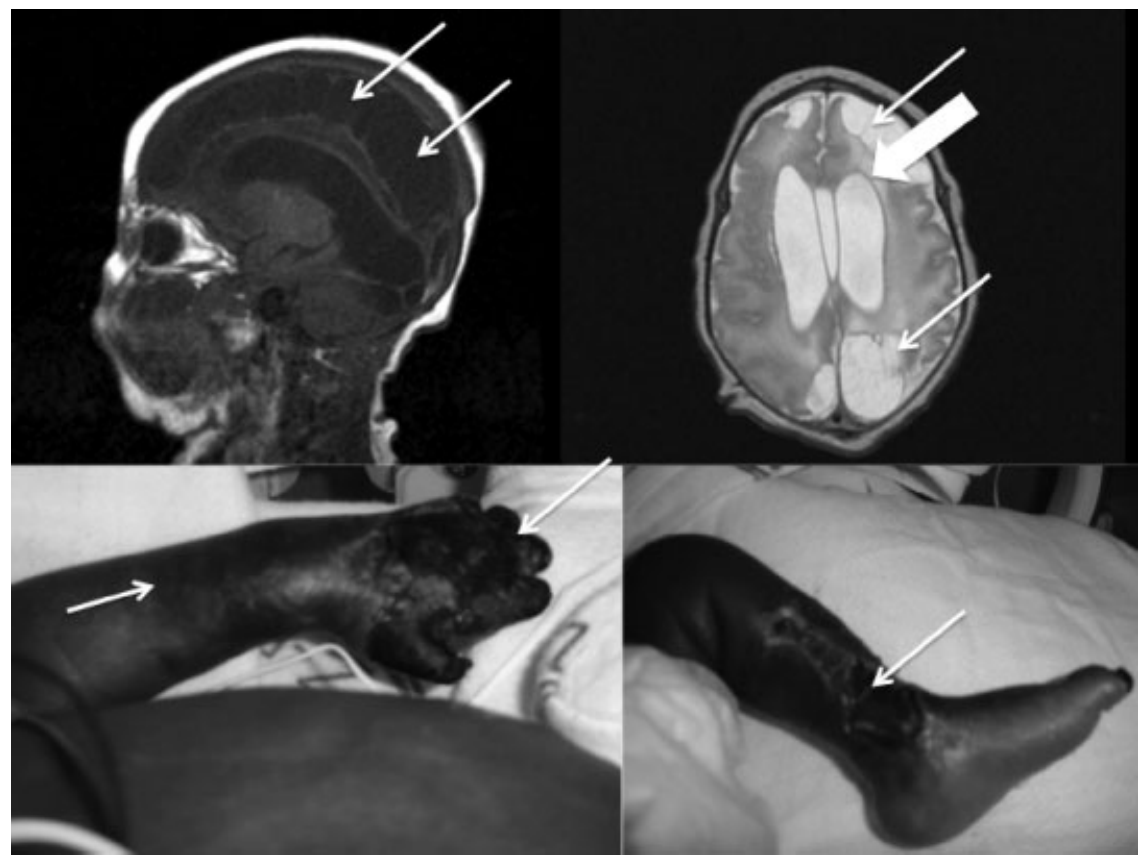

Fig. 1 Left upper panel: Magnetic resonance imaging of brain, sagittal view, showing extensive encephalomalacia (thin arrows). Right upper panel: Magnetic resonance imaging of brain, coronal view, showing cystic encephalomalacia of left frontoparietal and occipital lobes (thin arrows) and periventricular leukomalacia of lateral ventricles bilaterally (thick arrows). Left lower panel: Photograph showing demarcation of healthy and ischemic skin (up arrow) with gangrene (down arrow) and auto amputation of the fingers due to PF. Right lower panel: Necrosis extending to the tendons (arrow) of lower leg due to PF. PF, purpura fulminans.

and gentamicin $3.5 \mathrm{mg} / \mathrm{kg} /$ dose every 24 hours), fluids and inotropic support (dopamine, dobutamine, and epinephrine). CBC revealed total white blood cell count of $2,700 / \mathrm{mm}^{3}$ (5\% segments and $12 \%$ bands), hemoglobin of $12.9 \mathrm{~g} / \mathrm{dL}$, and platelets of $177,000 / \mathrm{mm}^{3}$. Within next 24 hours, the infant developed purplish discoloration of the left upper and lower extremities, which rapidly progressed to gangrenous skin lesions suggestive of PF. Coagulation studies, were normal with prothrombin time of 19.2 seconds, partial thromboplastin time of 31 seconds, and fibrinogen of $767 \mathrm{mg} / \mathrm{dL}$. Gram stain of CSF showed numerous gram-positive cocci in chains. Blood, CSF, and urine cultures were reported positive for GBS at 11,32 , and 33 hours of incubation, respectively. The isolate was further identified as serotype Ia (performed at the laboratories of the Centers for Disease Control and Prevention, Atlanta).

After CSF culture results, IV vancomycin was discontinued and IV crystalline penicillin G, 100,000 units/ $\mathrm{kg} /$ dose every 6 hours, was started and continued for 3 weeks. IV gentamicin was continued for 7 days. Repeat blood and CSF cultures were negative. Over the next 2 weeks, necrosis continued to worsen leading to autoamputation of all fingers of the left hand ( - Fig. 1, left lower panel) and all toes of the left leg. On day 29 , repeat head ultrasound study showed grade III hemorrhage in the left ventricle. On day 30 , the infant developed seizures involving both upper arms, which was confirmed by electroencephalogram. Seizures were managed with midazolam and phenobarbital. Repeat coagulation studies were normal at this time. On day 58, magnetic resonance imaging of brain showed cystic encephalomalacia involving bilateral frontal, parietal, and occipital lobes. Cystic periventricular leukomalacia was also noted around both lateral ventricles (-Fig. 1, upper panel).

The infant was discharged home at 4 months; he subsequently required ventriculoperitoneal shunt for hydrocephalus at 7 months. He had severe developmental delay and died at 9 months because of cardiopulmonary arrest of unknown etiology. The other twin survived and had no evidence of GBS infection. However, he was given a 10-day course of IV penicillin $\mathrm{G}$ as a preventive measure.

\section{Discussion}

We present a case of premature infant who developed severe PF secondary to late onset GBS sepsis and meningitis leading to autoamputation of digits and severe neurologic injury. Only a few cases of infectious PF in neonates have been reported thus so far. Lynn et al, ${ }^{1}$ Hon et al ${ }^{2}$, Zenciroglu et al, ${ }^{3}$ and Albarrak and Al-Matary ${ }^{4}$ have described PF in newborns due to early onset GBS, whereas Issacman et al ${ }^{5}$ have reported PF due to late onset GBS. However, these were cases of full-term infants whereas our case was an extremely premature infant. Stewart et $\mathrm{al}^{6}$ reported a preterm neonate with PF presumed to be of infectious origin, but cultures were negative and protein $\mathrm{C}$ and protein $\mathrm{S}$ were normal. Church et $\mathrm{al}^{7}$ reported a late preterm infant with PF secondary to Escherichia coli septicemia.

$\mathrm{PF}$ is classified as congenital, acquired, and idiopathic. Congenital causes are protein $C$ and protein $S$ deficiency that can result in thrombosis and PF within 72 hours of birth. ${ }^{8}$ Acquired causes are infections, disseminated intravascular coagulation, acute venous thrombosis, warfarin, galactosemia, and congenital heart disease. Idiopathic PF is rare 
and usually reported in older children. The leading cause of acquired PF in neonates is infection, among which GBS sepsis is the most common pathogen, although Staphylococcus aureus, Neisseria meningitidis, Escherichia coli, Enterobacter species, and Pseudomonas species also have been described.

The GBS strain from our patient was identified as serotype Ia, which is one of the most common serotypes in early and late onset neonatal sepsis in the United States. ${ }^{9,10}$ Martins et al ${ }^{11}$ found that serotypes Ia and III were the most common isolates for invasive infections in newborns. However, there is no preponderance of a specific serotype causing PF when compared to the overall serotype distribution of invasive GBS isolates. Consistent with findings of other studies, intrapartum GBS chemoprophylaxis did not prevent late onset GBS infection in our patient. Protein $C$ and protein $S$ were not known in our patient, but there was no family history of bleeding or coagulation disorders and the other twin did not have any manifestations. Also, PF occurred much later in life in our index case, which may suggest against congenital protein $\mathrm{C}$ and protein $\mathrm{S}$ deficiency

Management of infectious PF is focused on maintaining tissue perfusion and antibiotics, which may be beneficial only to some extent. Although antibiotics were initiated early in our patient, the disease progressed rapidly leading to devastating damage. Issacman et $\mathrm{al}^{5}$ used heparin to treat PF with some improvement. We could not use heparin in our index case because of grade III intraventricular hemorrhage (IVH).

In summary, our case highlights the rare presentation of severe sequelae due to PF secondary to GBS infection in a premature infant.

\section{Conflict of Interest}

The authors declare no conflict of interest.

\section{References}

1 Lynn NJ, Pauly TH, Desai NS. Purpura fulminans in three cases of early-onset neonatal group B streptococcal meningitis. J Perinatol 1991;11(2):144-146

2 Hon KL, So KW, Wong W, Cheung KL. Spot diagnosis: An ominous rash in a newborn. Ital J Pediatr 2009;35(1):10

3 Zenciroglu A, Karagol BS, Ipek MS, Okumus N, Yarali N, Aydin M. Neonatal purpura fulminans secondary to group B streptococcal infection. Pediatr Hematol Oncol 2010;27(8):620-625

4 Albarrak M, Al-Matary A. Neonatal purpura fulminans manifestation in early-onset group B Streptococcal infection. Am J Case Rep 2013;14:315-317

5 Issacman SH, Heroman WM, Lightsey AL. Purpura fulminans following late-onset group B beta-hemolytic streptococcal sepsis. Am J Dis Child 1984;138(10):915-916

6 Stewart FJ, McClure BG, Mayne E. Successful treatment of neonatal purpura fulminans with epoprostenol. J R Soc Med 1991;84(10): 623-624

7 Church J, Haram NH, Jones I, Hartnoll G. Purpura fulminans in an acute preterm neonate. Arch Dis Child Fetal Neonatal Ed 2013; 98(1):F76-F77

8 Marlar RA, Neumann A. Neonatal purpura fulminans due to homozygous protein $C$ or protein $S$ deficiencies. Semin Thromb Hemost 1990;16(4):299-309

9 Phares CR, Lynfield R, Farley MM, et al; Active Bacterial Core surveillance/Emerging Infections Program Network. Epidemiology of invasive group B streptococcal disease in the United States, 1999-2005. JAMA 2008;299(17):2056-2065

10 Bohnsack JF, Whiting A, Gottschalk M, et al. Population structure of invasive and colonizing strains of Streptococcus agalactiae from neonates of six U.S. Academic Centers from 1995 to 1999. J Clin Microbiol 2008;46(4):1285-1291

11 Martins ER, Andreu A, Correia P, et al; Microbiologist Group for the Study of Vertical Transmission Infections from the Catalan Society for Clinical Microbiology and Infectious Diseases. Group B streptococci causing neonatal infections in barcelona are a stable clonal population: 18-year surveillance. J Clin Microbiol 2011;49(8): 2911-2918 